\title{
FINITE-DIMENSIONAL ALGEBRAS AND QUIVERS
}

\author{
ALISTAIR SAVAGE
}

\begin{abstract}
This is an overview article on finite-dimensional algebras and quivers, written for the Encyclopedia of Mathematical Physics. We cover path algebras, Ringel-Hall algebras and the quiver varieties of Lusztig and Nakajima.
\end{abstract}

\section{INTRODUCTION}

Algebras and their representations are ubiquitous in mathematics. It turns out that representations of finite-dimensional algebras are intimately related to quivers, which are simply oriented graphs. Quivers arise naturally in many areas of mathematics, including representation theory, algebraic and differential geometry, Kac-Moody algebras, and quantum groups. In this article, we give a brief overview of some of these topics. We start by giving the basic definitions of associative algebras and their representations. We then introduce quivers and their representation theory, mentioning the connection to the representation theory of associative algebras. We also discuss in some detail the relationship between quivers and the theory of Lie algebras.

\section{Associative Algebras}

An algebra is a vector space $A$ over a field $k$ equipped with a multiplication which is distributive and such that

$$
a(x y)=(a x) y=x(a y), \forall a \in k, x, y \in A .
$$

When we wish to make the field explicit, we call $A$ a $k$-algebra. An algebra is associative if $(x y) z=x(y z)$ for all $x, y, z \in A$. $A$ has a unit, or multiplicative identity, if it contains an element $\mathbf{1}_{A}$ such that $\mathbf{1}_{A} x=x \mathbf{1}_{A}=x$ for all $x \in A$. From now on, we will assume all algebras are associative with unit. $A$ is said to be commutative if $x y=y x$ for all $x, y \in A$ and finite-dimensional if the underlying vector space of $A$ is finite-dimensional.

A vector subspace $I$ of $A$ is called a left (resp. right) ideal if $x y \in I$ for all $x \in A, y \in I$ (resp. $x \in I$, $y \in A$ ). If $I$ is both a right and a left ideal, it is called a two-sided ideal of $A$. If $I$ is a two-sided ideal of $A$, then the factor space $A / I$ is again an algebra.

An algebra homomorphism is a linear map $f$ : $A_{1} \rightarrow A_{2}$ between two algebras such that

$$
\begin{gathered}
f\left(\mathbf{1}_{A_{1}}\right)=\mathbf{1}_{A_{2}}, \text { and } \\
f(x y)=f(x) f(y), \forall x, y \in A .
\end{gathered}
$$

A representation of an algebra $A$ is an algebra homomorphism $\rho: A \rightarrow \operatorname{End}_{k}(V)$ for a $k$-vector space $V$. Here $\operatorname{End}_{k}(V)$ is the space of endomorphisms of the vector space $V$ with multiplication given by composition. Given a representation of an algebra $A$ on a vector space $V$, we may view $V$ as an $A$-module with the action of $A$ on $V$ given by

$$
a \cdot v=\rho(a) v, a \in A, v \in V .
$$

A morphism $\psi: V \rightarrow W$ of two $A$-modules (or equivalently, representations of $A$ ), is a linear map commuting with the action of $A$. That is, it is a linear map satisfying

$$
a \cdot \psi(v)=\psi(a \cdot v), \quad \forall a \in A, v \in V .
$$

Let $G$ be a commutative monoid (a set with an associative multiplication and a unit element). A $G$-graded $k$-algebra is a $k$-algebra which can be expressed as a direct sum $A=\oplus_{g \in G} A_{g}$ such that $a A_{g} \subset A_{g}$ for all $a \in k$ and $A_{g_{1}} A_{g_{2}} \subset A_{g_{1}+g_{2}}$ for all $g_{1}, g_{2} \in G$. A morphism $\psi: A \rightarrow B$ of $G$-graded algebras is a $k$-algebra morphism respecting the grading, that is, satisfying $\psi\left(A_{g}\right) \subset B_{g}$ for all $g \in G$.

Date: May 4, 2005.

2000 Mathematics Subject Classification. 16G20,16-02.

This research was supported in part by the Natural Sciences and Engineering Research Council (NSERC) of Canada. 


\section{Quivers AND PATH ALGEBRAS}

A quiver is simply an oriented graph. More precisely, a quiver is a pair $Q=\left(Q_{0}, Q_{1}\right)$ where $Q_{0}$ is a finite set of vertices and $Q_{1}$ is a finite set of arrows (oriented edges) between them. For $a \in Q_{1}$, we let $h(a)$ denote the head of $a$ and $t(a)$ denote the tail of $a$. A path in $Q$ is a sequence $x=\rho_{1} \rho_{2} \ldots \rho_{m}$ of arrows such that $h\left(\rho_{i+1}\right)=t\left(\rho_{i}\right)$ for $1 \leq i \leq m-1$. We let $t(x)=t\left(\rho_{m}\right)$ and $h(\rho)=h\left(\rho_{1}\right)$ denote the initial and final vertices of the path $x$. For each vertex $i \in Q_{0}$, we let $e_{i}$ denote the trivial path which starts and ends at the vertex $i$.

Fix a field $k$. The path algebra $k Q$ associated to a quiver $Q$ is the $k$-algebra whose underlying vector space has basis the set of paths in $Q$, and with the product of paths given by concatenation. Thus, if $x=\rho_{1} \ldots \rho_{m}$ and $y=\sigma_{1} \ldots \sigma_{n}$ are two paths, then $x y=\rho_{1} \ldots \rho_{m} \sigma_{1} \ldots \sigma_{n}$ if $h(y)=t(x)$ and $x y=0$ otherwise. We also have

$$
\begin{gathered}
e_{i} e_{j}=\left\{\begin{array}{l}
e_{i} \text { if } i=j \\
0 \text { if } i \neq j
\end{array},\right. \\
e_{i} x=\left\{\begin{array}{l}
x \text { if } h(x)=i \\
0 \text { if } h(x) \neq i
\end{array},\right. \\
x e_{i}=\left\{\begin{array}{l}
x \text { if } t(x)=i \\
0 \text { if } t(x) \neq i
\end{array},\right.
\end{gathered}
$$

for $x \in k Q$. This multiplication is associative. Note that $e_{i} A$ and $A e_{i}$ have bases given by the set of paths ending and starting at $i$ respectively. The path algebra has a unit given by $\sum_{i \in Q_{0}} e_{i}$.

Example 3.1. Let $Q$ be the following quiver.

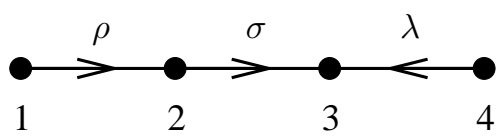

Then $k Q$ has a basis given by the set of paths $\left\{e_{1}, e_{2}, e_{3}, e_{4}, \rho, \sigma, \lambda, \sigma \rho\right\}$. Some sample products are $\rho \sigma=0, \lambda \lambda=0, \lambda \sigma=0, e_{3} \sigma=\sigma e_{2}=\sigma, e_{2} \sigma=0$.

Example 3.2. Let $Q$ be the following quiver (the socalled Jordan quiver).

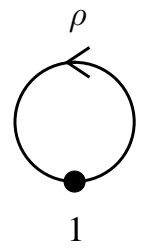

Then $k Q \cong k[t]$, the algebra of polynomials in one variable.
Note that the path algebra $k Q$ is finite-dimensional if and only if $Q$ has no oriented cycles (paths with the same head and tail vertex).

Example 3.3. Let $Q$ be the following quiver.

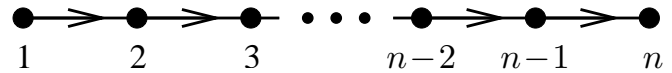

Then for every $1 \leq i \leq j \leq n$, there is a unique path from $i$ to $j$. Let $f: k Q \rightarrow M_{n}(k)$ be the linear map from the path algebra to the $n \times n$ matrices with entries in the field $k$ that sends the unique path from $i$ to $j$ to the matrix $E_{j i}$ with $(j, i)$ entry 1 and all other entries zero. Then one can show that $f$ is an isomorphism onto the algebra of lower triangular matrices.

\section{Representations of QUivers}

Fix a field $k$. A representation of a quiver $Q$ is an assignment of a vector space to each vertex and to each arrow a linear map between the vector spaces assigned to its tail and head. More precisely, a representation $V$ of $Q$ is a collection

$$
\left\{V_{i} \mid i \in Q_{0}\right\}
$$

of finite-dimensional $k$-vector spaces together with a collection

$$
\left\{V_{\rho}: V_{t(\rho)} \rightarrow V_{h(\rho)} \mid \rho \in Q_{1}\right\}
$$

of $k$-linear maps. Note that a representation $V$ of a quiver $Q$ is equivalent to a representation of the path algebra $k Q$. The dimension of $V$ is the map $d_{V}: Q_{0} \rightarrow \mathbb{Z}_{>0}$ given by $d_{V}(i)=\operatorname{dim} V_{i}$ for $i \in Q_{0}$.

If $V$ and $W$ are two representations of a quiver $Q$, then a morphism $\psi: V \rightarrow W$ is a collection of $k$-linear maps

$$
\left\{\psi_{i}: V_{i} \rightarrow W_{i} \mid i \in Q_{0}\right\}
$$

such that

$$
W_{\rho} \psi_{t(\rho)}=\psi_{h(\rho)} V_{\rho}, \forall \rho \in Q_{1} .
$$

Proposition 4.1. Let $A$ be a finite-dimensional $k$ algebra. Then the category of representations of $A$ is equivalent to the category of representations of the algebra $k Q / I$ for some quiver $Q$ and some two-sided ideal $I$ of $k Q$.

It is for this reason that the study of finitedimensional associative algebras is intimately related to the study of quivers.

We define the direct sum $V \oplus W$ of two representations $V$ and $W$ of a quiver $Q$ by

$$
(V \oplus W)_{i}=V_{i} \oplus W_{i}, i \in Q_{0}
$$

and $(V \oplus W)_{\rho}: V_{t(\rho)} \oplus W_{t(\rho)} \rightarrow V_{h(\rho)} \oplus W_{h(\rho)}$ by

$$
(V \oplus W)_{\rho}((v, w))=\left(V_{\rho}(v), W_{\rho}(w)\right)
$$


for $v \in V_{t(\rho)}, w \in W_{t(\rho)}, \rho \in Q_{1}$. A representation $V$ is trivial if $V_{i}=0$ for all $i \in Q_{0}$ and simple if its only subrepresentations are the zero representation and $V$ itself. We say that $V$ is decomposable if it is isomorphic to $W \oplus U$ for some nontrivial representations $W$ and $U$. Otherwise, we call $V$ indecomposable. Every representation of a quiver has a decomposition into indecomposable representations that is unique up to isomorphism and permutation of the components. Thus, to classify all representations of a quiver, it suffices to classify the indecomposable representations.

Example 4.2. Let $Q$ be the following quiver.

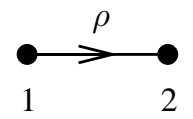

Then $Q$ has three indecomposable representations $U$, $V$ and $W$ given by:

$$
\begin{gathered}
U_{1}=k, U_{2}=0, U_{\rho}=0, \\
V_{1}=0, V_{2}=k, V_{\rho}=0, \\
W_{1}=k, W_{2}=k, W_{\rho}=1 .
\end{gathered}
$$

Then any representation $Z$ of $Q$ is isomorphic to

$$
Z \cong U^{d_{1}-r} \oplus V^{d_{2}-r} \oplus W^{r},
$$

where $d_{1}=\operatorname{dim} Z_{1}, d_{2}=\operatorname{dim} Z_{2}, r=\operatorname{rank} Z_{\rho}$.

Example 4.3. Let $Q$ be the Jordan quiver. Then representations $V$ of $Q$ are classified up to isomorphism by the Jordan normal form of $V_{\rho}$ where $\rho$ is the single arrow of the quiver. Indecomposable representations correspond to single Jordan blocks. These are parametrized by a discrete parameter $n$ (the size of the block) and a continuous parameter $\lambda$ (the eigenvalue of the block).

A quiver is said to be of finite type if it has only finitely many indecomposable representations (up to isomorphism). If a quiver has infinitely many isomorphism classes but they can be split into families, each parametrized by a single continuous parameter, then we say the quiver is of tame (or affine) type. If a quiver is of neither finite nor tame type, it is of wild type. It turns out that there is a rather remarkable relationship between the classification of quivers and their representations and the theory of Kac-Moody algebras.

The Euler form or Ringel form of a quiver $Q$ is defined to be the asymmetric bilinear form on $\mathbb{Z}^{Q_{0}}$ given by

$$
\langle\alpha, \beta\rangle=\sum_{i \in Q_{0}} \alpha(i) \beta(i)-\sum_{\rho \in Q_{1}} \alpha(t(\rho)) \beta(h(\rho)) .
$$

In the standard coordinate basis of $\mathbb{Z}^{Q_{0}}$, the Euler form is represented by the matrix $E=\left(a_{i j}\right)$ where

$$
a_{i j}=\delta_{i j}-\#\left\{\rho \in Q_{1} \mid t(\rho)=i, h(\rho)=j\right\} .
$$

Here $\delta_{i j}$ is the Kronecker delta symbol. We define the Cartan form of the quiver $Q$ to be the symmetric bilinear form given by

$$
(\alpha, \beta)=\langle\alpha, \beta\rangle+\langle\beta, \alpha\rangle .
$$

Note that the Cartan form is independent of the orientation of the arrows in $Q$. In the standard coordinate basis of $\mathbb{Z}^{Q_{0}}$, the Cartan form is represented by the Cartan matrix $C=\left(c_{i j}\right)$ where $c_{i j}=a_{i j}+a_{j i}$.

Example 4.4. For the quiver in Example 3.1, the Euler matrix is

$$
E=\left(\begin{array}{cccc}
1 & -1 & 0 & 0 \\
0 & 1 & -1 & 0 \\
0 & 0 & 1 & 0 \\
0 & 0 & -1 & 1
\end{array}\right)
$$

and the Cartan matrix is

$$
C=\left(\begin{array}{cccc}
2 & -1 & 0 & 0 \\
-1 & 2 & -1 & 0 \\
0 & -1 & 2 & -1 \\
0 & 0 & -1 & 2
\end{array}\right)
$$

The Tits form $q$ of a quiver $Q$ is defined by

$$
q(\alpha)=\langle\alpha, \alpha\rangle=\frac{1}{2}(\alpha, \alpha) .
$$

It is known that the number of continuous parameters describing representations of dimension $\alpha$ for $\alpha \neq 0$ is greater than or equal to $1-q(\alpha)$.

Let $\mathfrak{g}$ be the Kac-Moody algebra associated to the Cartan matrix of a quiver $Q$. By forgetting the orientation of the arrows of $Q$, we obtain the underlying (undirected) graph. This is the Dynkin graph of $\mathfrak{g}$. Associated to $\mathfrak{g}$ is a root system and a set of simple roots $\left\{\alpha_{i} \mid i \in Q_{0}\right\}$ indexed by the vertices of the Dynkin graph.

Theorem 4.5 (Gabriel's Theorem).

(1) A quiver is of finite type it and only if the underlying graph is a union of Dynkin graphs of type $A, D$, or $E$.

(2) A quiver is of tame type if and only if the underlying graph is a union of Dynkin graphs of type $A, D$, or $E$ and extended Dynkin graphs of type $\widehat{A}, \widehat{D}$, or $\widehat{E}$ (with at least one extended Dynkin graph).

(3) The isomorphism classes of indecomposable representations of a quiver $Q$ of finite type are in one-to-one correspondence with the positive roots of the root system associated to the 
underlying graph of $Q$. The correspondence is given by

$$
V \mapsto \sum_{i \in Q_{0}} d_{V}(i) \alpha_{i}
$$

The Dynkin graphs of type $A, D$, and $E$ are as follows.

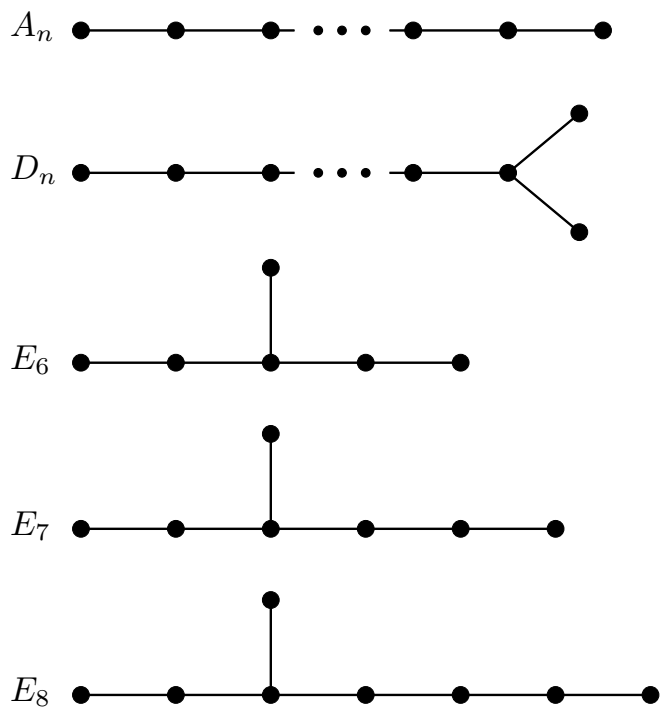

Here the subscript indicates the number of vertices in the graph.

The extended Dynkin graphs of type $\widehat{A}, \widehat{D}$, and $\widehat{E}$ are as follows.
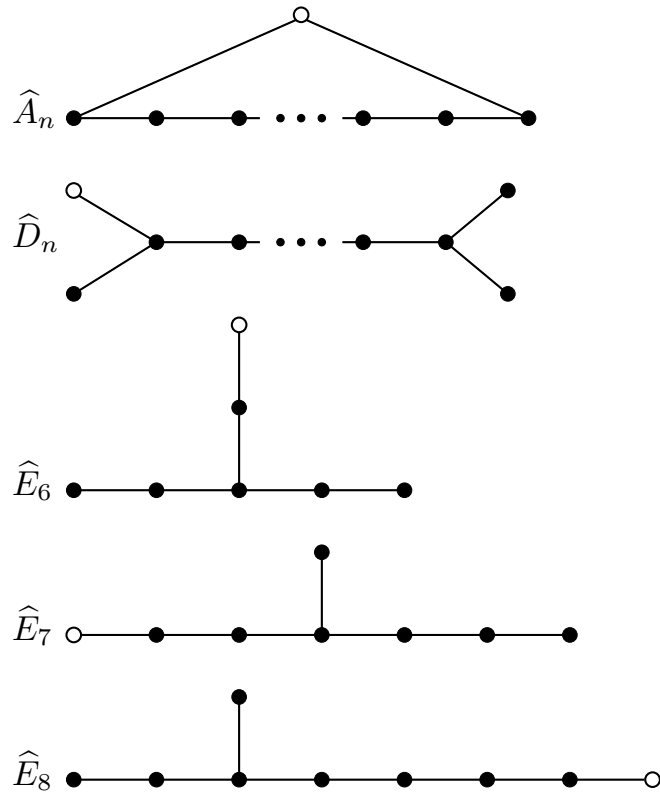

Here we have used an open dot to denote the vertex that was added to the corresponding Dynkin graph of type $A, D$, or $E$.
Theorem 4.6 (Kac's Theorem). Let $Q$ be an arbitrary quiver. The dimension vectors of indecomposable representations of $Q$ correspond to positive roots of the root system associated to the underlying graph of $Q$ (and are thus independent of the orientation of the arrows of $Q$ ). The correspondence is given by

$$
d_{V} \mapsto \sum_{i \in Q_{0}} d_{V}(i) \alpha_{i}
$$

Note that in Kac's Theorem, it is not asserted that the isomorphism classes are in one-to-one correspondence with the roots as in the finite case considered in Gabriel's theorem. It turns out that in the general case, dimension vectors for which there is exactly one isomorphism class correspond to real roots while imaginary roots correspond to dimension vectors for which there are families of representations.

Example 4.7. Let $Q$ be the quiver of type $A_{n}$, oriented as follows.

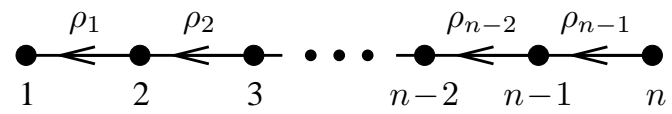

It is known that the set of positive roots of the simple Lie algebra of type $A_{n}$ is

$$
\left\{\sum_{i=j}^{l} \alpha_{i} \mid 1 \leq j \leq l \leq n\right\} \sqcup\{0\} .
$$

The zero root corresponds to the trivial representation. The root $\sum_{i=j}^{l} \alpha_{i}$ for some $1 \leq j \leq l \leq n$ corresponds to the unique (up to isomorphism) representation $V$ with

$$
V_{i}=\left\{\begin{array}{l}
k \text { if } j \leq i \leq l \\
0 \text { otherwise }
\end{array}\right.
$$

and

$$
V_{\rho_{i}}=\left\{\begin{array}{l}
1 \text { if } j \leq i \leq l-1 \\
0 \text { otherwise }
\end{array} .\right.
$$

Example 4.8. Let $Q$ be the quiver of type $\widehat{A}_{n}$, with all arrows oriented in the same direction (for instance, counter-clockwise). The positive root $\sum_{i=0}^{n} \alpha_{i}$ (where $\{0,1,2, \ldots, n\}$ are the vertices of the quiver) is imaginary. There is a one parameter family of isomorphism classes of indecomposable representations where the maps assigned to each arrow are non-zero. The parameter is the composition of the maps around the loop.

If a quiver $Q$ has no oriented cycles, then the only simple $k Q$-modules are the modules $S^{i}$ for $i \in Q_{0}$ where

$$
S_{j}^{i}=\left\{\begin{array}{l}
k \text { if } i=j \\
0 \text { if } i \neq j
\end{array}\right.
$$

and $S_{\rho}^{i}=0$ for all $\rho \in Q_{1}$. 


\section{Ringel-Hall Algebras}

Let $k$ be the finite field $\mathbb{F}_{q}$ with $q$ elements and let $Q$ be a quiver with no oriented cycles. Let $\mathcal{P}$ be the set of all isomorphism classes of $k Q$-modules which are finite as sets (since $k$ is finite-dimensional, these are just the quiver representations we considered above). Let $A$ be a commutative integral domain containing $\mathbb{Z}$ and elements $v, v^{-1}$ such that $v^{2}=q$. The Ringel-Hall algebra $H=H_{A, v}(k Q)$ is the free $A$ module with basis $\{[V]\}$ indexed by the isomorphism classes of representations of the quiver $Q$, with an $A$-bilinear multiplication defined by

$$
\left[V^{1}\right] \cdot\left[V^{2}\right]=v^{\left\langle\operatorname{dim} V^{1}, \operatorname{dim} V^{2}\right\rangle} \sum_{V} g_{V^{1}, V^{2}}^{V}[V] .
$$

Here $\left\langle\operatorname{dim} V^{1}, \operatorname{dim} V^{2}\right\rangle$ is the Euler form and $g_{V^{1}, V^{2}}^{V}$ is the number of submodules $W$ of $V$ such that $V / W \cong V^{1}$ and $W \cong V^{2}$. $H$ is an associative $\mathbb{Z}_{\geq 0^{-}}^{Q_{0}}$ graded algebra, with identity element [0], the isomorphism class of the trivial representation. The grading $H=\oplus_{\alpha} H_{\alpha}$ is given by letting $H_{\alpha}$ be the $A$ span of the set of isomorphism classes $[V]$ such that $\operatorname{dim} V=\alpha$.

Let $C=C_{A, v}(k Q)$ be the $A$-subalgebra of $H$ generated by the isomorphism classes $\left[S^{i}\right]$ of the simple $k Q$-modules. $C$ is called the composition algebra. If the underlying graph of $Q$ is of finite type, then $C=H$.

Now let $\mathcal{K}$ be a set of finite fields $k$ such that the set $\{|k| \mid k \in \mathcal{K}\}$ is infinite. Let $A$ be an integral domain containing $\mathbb{Q}$ and, for each $k \in \mathcal{K}$, an element $v_{k}$ such that $v_{k}^{2}=|k|$. For each $k \in \mathcal{K}$ we have the corresponding composition algebra $C_{k}$, generated by the elements $\left[{ }^{k} S^{i}\right]$ (here we make the field $k$ explicit). Now let $C$ be the subring of $\prod_{k \in \mathcal{K}} C_{k}$ generated by $\mathbb{Q}$ and the elements

$$
\begin{gathered}
t=\left(t_{k}\right)_{k \in \mathcal{K}}, t_{k}=v_{k}, \\
t^{-1}=\left(t_{k}^{-1}\right)_{k \in \mathcal{K}}, t_{k}^{-1}=\left(v^{k}\right)^{-1}, \\
u^{i}=\left(u_{k}^{i}\right)_{k \in \mathcal{K}}, u_{k}^{i}=\left[{ }^{k} S^{i}\right], i \in Q_{0} .
\end{gathered}
$$

Now, $t$ lies in the center of $C$ and if $p(t)=0$ for some polynomial $p$, then $p$ must be the zero polynomial since the set of $v_{k}$ is infinite. Thus we may think of $C$ as the $A$-algebra generated by the $u^{i}$, $i \in Q_{0}$, with $A=\mathbb{Q}\left[t, t^{-1}\right]$ and $t$ an indeterminate. Let $C^{*}=\mathbb{Q}(t) \otimes_{A} C$. We call $C^{*}$ the generic composition algebra.

Let $\mathfrak{g}$ be the Kac-Moody algebra associated to the Cartan matrix of the quiver $Q$ and let $U$ be the quantum group associated by Drinfeld and Jimbo to g. It has a triangular decomposition $U=U^{-} \otimes U^{0} \otimes U^{+}$. Specifically, $U^{+}$is the $\mathbb{Q}(t)$-algebra with generators
$E_{i}, i \in Q_{0}$ and relations

$$
\sum_{p=0}^{1-c_{i j}}(-1)^{p}\left[\begin{array}{c}
1-c_{i j} \\
p
\end{array}\right] E_{i}^{p} E_{j} E_{i}^{1-c_{i j}-p}, i \neq j,
$$

where $c_{i j}$ are the entries of the Cartan matrix and

$$
\begin{gathered}
{\left[\begin{array}{c}
m \\
p
\end{array}\right]=\frac{[m] !}{[p] ![m-p] !}} \\
{[n]=\frac{t^{n}-t^{-n}}{t-t^{-1}},[n] !=[1][2] \ldots[n] .}
\end{gathered}
$$

Theorem 5.1. There is a $\mathbb{Q}(t)$-algebra isomorphism $C^{*} \rightarrow U^{+}$sending $u_{i} \mapsto E_{i}$ for all $i \in Q_{0}$.

The proof of Theorem [5.1] is due to Ringel in the case that the underlying graph of $Q$ is of finite or affine type. The more general case presented here is due to Green.

All of the Kac-Moody algebras considered so far have been simply-laced. That is, their Cartan matrices are symmetric. There is a way to deal with non-simply-laced Kac-Moody algebras using species. We will not treat this subject in this article.

\section{Quiver Varieties}

One can use varieties associated to quivers to yield a geometric realization of the upper half of the universal enveloping algebra of a Kac-Moody algebra $\mathfrak{g}$ and its irreducible highest-weight representations.

6.1. Lusztig's Quiver Varieties. We first introduce the quiver varieties, first defined by Lusztig, which yield a geometric realization of the upper half $\mathcal{U}^{+}$of the universal enveloping algebra of a simplylaced Kac-Moody algebra $\mathfrak{g}$. Let $Q=\left(Q_{0}, Q_{1}\right)$ be the quiver whose vertices $Q_{0}$ are the vertices of the Dynkin diagram of $\mathfrak{g}$ and whose set of arrows $Q_{1}$ consists of all the edges of the Dynkin diagram with both orientations. By definition, $\mathcal{U}^{+}$is the $\mathbb{Q}$-algebra defined by generators $e_{i}, i \in Q_{0}$, subject to the Serre relations

$$
\sum_{p=0}^{1-c_{i j}}(-1)^{p}\left(\begin{array}{c}
1-c_{i j} \\
p
\end{array}\right) e_{i}^{p} e_{j} e^{1-c_{i j}-p}=0
$$

for all $i \neq j$ in $Q_{0}$, where $c_{i j}$ are the entries of the Car$\tan$ matrix associated to $Q$. For any $\nu=\sum_{i \in Q_{0}} \nu_{i} i$, $\nu_{i} \in \mathbb{N}$, let $\mathcal{U}_{\nu}^{+}$be the subspace of $\mathcal{U}^{+}$generated by the monomials $e_{i_{1}} e_{i_{2}} \ldots e_{i_{n}}$ for various sequences $i_{1}, i_{2}, \ldots, i_{n}$ in which $i$ appears $\nu_{i}$ times for each $i \in Q_{0}$. Thus $\mathcal{U}^{+}=\oplus_{\nu} \mathcal{U}_{\nu}^{+}$. Let $\mathcal{U}_{\mathbb{Z}}^{+}$be the subring of $\mathcal{U}^{+}$generated by the elements $e_{i}^{p} / p$ ! for $i \in Q_{0}$, $p \in \mathbb{N}$. Then $\mathcal{U}_{\mathbb{Z}}^{+}=\oplus_{\nu} \mathcal{U}_{\mathbb{Z}, \nu}^{+}$where $\mathcal{U}_{\mathbb{Z}, \nu}^{+}=\mathcal{U}_{\mathbb{Z}}^{+} \cap \mathcal{U}_{\nu}^{+}$.

We define the involution ${ }^{-}: Q_{1} \rightarrow Q_{1}$ to be the function which takes $\rho \in Q_{1}$ to the element of $Q_{1}$ consisting of the same edge with opposite orientation. 
An orientation of our graph/quiver is a choice of a subset $\Omega \subset Q_{1}$ such that $\Omega \cup \bar{\Omega}=Q_{1}$ and $\Omega \cap \bar{\Omega}=\emptyset$.

Let $\mathcal{V}$ be the category of finite-dimensional $Q_{0^{-}}$ graded vector spaces $\mathbf{V}=\oplus_{i \in Q_{0}} \mathbf{V}_{i}$ over $\mathbb{C}$ with morphisms being linear maps respecting the grading. Then $\mathbf{V} \in \mathcal{V}$ shall denote that $\mathbf{V}$ is an object of $\mathcal{V}$. The dimension of $\mathbf{V} \in \mathcal{V}$ is given by $\mathbf{v}=\operatorname{dim} \mathbf{V}=\left(\operatorname{dim} \mathbf{V}_{0}, \ldots, \operatorname{dim} \mathbf{V}_{n}\right)$.

Given $\mathbf{V} \in \mathcal{V}$, let $E_{V}$ be the space of representations of $Q$ with underlying vector space $\mathbf{V}$. That is,

$$
\mathbf{E}_{\mathbf{V}}=\bigoplus_{\rho \in Q_{1}} \operatorname{Hom}\left(\mathbf{V}_{t(\rho)}, \mathbf{V}_{h(\rho)}\right)
$$

For any subset $Q_{1}^{\prime}$ of $Q_{1}$, let $\mathbf{E}_{\mathbf{V}, Q_{1}^{\prime}}$ be the subspace of $\mathbf{E}_{\mathbf{V}}$ consisting of all vectors $x=\left(x_{\rho}\right)$ such that $x_{\rho}=0$ whenever $\rho \notin Q_{1}^{\prime}$. The algebraic group $G_{\mathbf{V}}=\prod_{i} \operatorname{Aut}\left(\mathbf{V}_{i}\right)$ acts on $\mathbf{E}_{\mathbf{V}}$ and $\mathbf{E}_{\mathbf{V}, Q_{1}^{\prime}}$ by

$$
(g, x)=\left(\left(g_{i}\right),\left(x_{\rho}\right)\right) \mapsto g x=\left(x_{\rho}^{\prime}\right)=\left(g_{h(\rho)} x_{\rho} g_{t(\rho)}^{-1}\right) .
$$

Define the function $\varepsilon: Q_{1} \rightarrow\{-1,1\}$ by $\varepsilon(\rho)=1$ for all $\rho \in \Omega$ and $\varepsilon(\rho)=-1$ for all $\rho \in \bar{\Omega}$. Let $\langle\cdot, \cdot\rangle$ be the nondegenerate, $G_{\mathbf{V}}$-invariant, symplectic form on $\mathbf{E}_{\mathbf{V}}$ with values in $\mathbb{C}$ defined by

$$
\langle x, y\rangle=\sum_{\rho \in Q_{1}} \varepsilon(\rho) \operatorname{tr}\left(x_{\rho} y_{\bar{\rho}}\right) .
$$

Note that $\mathbf{E}_{\mathbf{V}}$ can be considered as the cotangent space of $\mathbf{E}_{\mathbf{V}, \Omega}$ under this form.

The moment map associated to the $G_{\mathbf{V}}$-action on the symplectic vector space $\mathbf{E}_{\mathbf{V}}$ is the map $\psi: \mathbf{E}_{\mathbf{V}} \rightarrow$ $\mathbf{g l}_{\mathbf{V}}=\prod_{i}$ End $\mathbf{V}_{i}$, the Lie algebra of $G L_{\mathbf{V}}$, with $i$ component $\psi_{i}: \mathbf{E}_{\mathbf{V}} \rightarrow$ End $\mathbf{V}_{i}$ given by

$$
\psi_{i}(x)=\sum_{\rho \in Q_{1}, h(\rho)=i} \varepsilon(\rho) x_{\rho} x_{\bar{\rho}} .
$$

Definition 6.1. An element $x \in \mathbf{E}_{\mathbf{V}}$ is said to be nilpotent if there exists an $N \geq 1$ such that for any sequence $\rho_{1}, \rho_{2}, \ldots, \rho_{N}$ in $H$ satisfying $t\left(\rho_{1}\right)=h\left(\rho_{2}\right)$, $t\left(\rho_{2}\right)=h\left(\rho_{3}\right), \ldots, t\left(\rho_{N-1}\right)=h\left(\rho_{N}\right)$, the composition $x_{\rho_{1}} x_{\rho_{2}} \ldots x_{\rho_{N}}: \mathbf{V}_{t\left(\rho_{N}\right)} \rightarrow \mathbf{V}_{h\left(\rho_{1}\right)}$ is zero.

Definition 6.2. Let $\Lambda_{\mathbf{V}}$ be the set of all nilpotent elements $x \in \mathbf{E}_{\mathbf{V}}$ such that $\psi_{i}(x)=0$ for all $i \in I$.

A subset of an algebraic variety is said to be constructible if it is obtained from subvarieties from a finite number of the usual set-theoretic operations. A function $f: A \rightarrow \mathbb{Q}$ on an algebraic variety $A$ is said to be a constructible function if $f^{-1}(a)$ is a constructible set for all $a \in \mathbb{Q}$ and is empty for all but finitely many $a$. Let $M\left(\Lambda_{\mathbf{V}}\right)$ denote the $\mathbb{Q}$ vector space of all constructible functions on $\Lambda_{\mathbf{V}}$. Let $\widetilde{M}\left(\Lambda_{\mathbf{V}}\right)$ denote the $\mathbb{Q}$-subspace of $M\left(\Lambda_{\mathbf{V}}\right)$ consisting of those functions that are constant on any $G_{\mathbf{V}}$-orbit in $\Lambda_{\mathbf{V}}$.
Let $\mathbf{V}, \mathbf{V}^{\prime}, \mathbf{V}^{\prime \prime} \in \mathcal{V}$ such that $\operatorname{dim} \mathbf{V}=\operatorname{dim} \mathbf{V}^{\prime}+$ $\operatorname{dim} \mathbf{V}^{\prime \prime}$. Now, suppose that $\mathbf{S}$ is an $I$-graded subspace of $\mathbf{V}$. For $x \in \Lambda_{\mathbf{V}}$ we say that $\mathbf{S}$ is $x$-stable if $x(\mathbf{S}) \subset$ S. Let $\Lambda_{\mathbf{V} ; \mathbf{V}^{\prime}, \mathbf{V}^{\prime \prime}}$ be the variety consisting of all pairs $(x, \mathbf{S})$ where $x \in \Lambda_{\mathbf{V}}$ and $\mathbf{S}$ is an $I$-graded $x$-stable subspace of $\mathbf{V}$ such that $\operatorname{dim} \mathbf{S}=\operatorname{dim} \mathbf{V}^{\prime \prime}$. Now, if we fix some isomorphisms $\mathbf{V} / \mathbf{S} \cong \mathbf{V}^{\prime}, \mathbf{S} \cong \mathbf{V}^{\prime \prime}$, then $x$ induces elements $x^{\prime} \in \Lambda_{\mathbf{V}^{\prime}}$ and $x^{\prime \prime} \in \Lambda_{\mathbf{V}^{\prime \prime}}$. We then have the maps

$$
\Lambda_{\mathbf{V}^{\prime}} \times \Lambda_{\mathbf{V}^{\prime \prime}} \stackrel{p_{1}}{\longleftarrow} \Lambda_{\mathbf{V} ; \mathbf{V}^{\prime}, \mathbf{V}^{\prime \prime}} \stackrel{p_{2}}{\longrightarrow} \Lambda_{\mathbf{V}}
$$

where $p_{1}(x, \mathbf{S})=\left(x^{\prime}, x^{\prime \prime}\right), p_{2}(x, \mathbf{S})=x$.

For a holomorphic map $\pi$ between complex varieties $A$ and $B$, let $\pi$ ! denote the map between the spaces of constructible functions on $A$ and $B$ given by

$$
\left(\pi_{!} f\right)(y)=\sum_{a \in \mathbb{Q}} a \chi\left(\pi^{-1}(y) \cap f^{-1}(a)\right) .
$$

Let $\pi^{*}$ be the pullback map from functions on $B$ to functions on $A$ acting as $\pi^{*} f(y)=f(\pi(y))$. We then define a map

$$
\widetilde{M}\left(\Lambda_{\mathbf{V}^{\prime}}\right) \times \widetilde{M}\left(\Lambda_{\mathbf{V}^{\prime \prime}}\right) \rightarrow \widetilde{M}\left(\Lambda_{\mathbf{V}}\right)
$$

by $\left(f^{\prime}, f^{\prime \prime}\right) \mapsto f^{\prime} * f^{\prime \prime}$ where

$$
f^{\prime} * f^{\prime \prime}=\left(p_{2}\right) ! p_{1}^{*}\left(f^{\prime} \times f^{\prime \prime}\right) .
$$

Here $f^{\prime} \times f^{\prime \prime} \in \widetilde{M}\left(\Lambda_{\mathbf{V}^{\prime}} \times \Lambda_{\mathbf{V}^{\prime \prime}}\right)$ is defined by $\left(f^{\prime} \times\right.$ $\left.f^{\prime \prime}\right)\left(x^{\prime}, x^{\prime \prime}\right)=f^{\prime}\left(x^{\prime}\right) f^{\prime \prime}\left(x^{\prime \prime}\right)$. The map (6.1) is bilinear and defines an associatve $\mathbb{Q}$-algebra structure on $\oplus_{\nu} \widetilde{M}\left(\Lambda_{\mathbf{V}^{\nu}}\right)$ where $\mathbf{V}^{\nu}$ is the object of $\mathcal{V}$ defined by $\mathbf{V}_{i}^{\nu}=\mathbb{C}^{\nu_{i}}$.

There is a unique algebra homomorphism $\kappa$ : $\mathcal{U}^{+} \rightarrow \oplus_{\nu} \widetilde{M}\left(\Lambda_{\mathbf{V}^{\nu}}\right)$ such that $\kappa\left(e_{i}\right)$ is the function on the point $\Lambda_{\mathbf{V}^{i}}$ with value 1 . Then $\kappa$ restricts to a map $\kappa_{\nu}: \mathcal{U}_{\nu}^{+} \rightarrow \widetilde{M}\left(\Lambda_{\mathbf{V}^{\nu}}\right)$. It can be shown that $\kappa_{p i}\left(e_{i}^{p} / p !\right)$ is the function 1 on the point $\Lambda_{\mathbf{V}^{p i}}$ for $i \in Q_{0}, p \in \mathbb{Z}_{\geq 0}$.

Let $\widetilde{M}_{\mathbb{Z}}\left(\Lambda_{\mathbf{V}}\right)$ be the set of all functions in $\widetilde{M}\left(\Lambda_{\mathbf{V}}\right)$ that take on only integer values. One can show that if $f^{\prime} \in \widetilde{M}_{\mathbb{Z}}\left(\Lambda_{\mathbf{V}^{\prime}}\right)$ and $f^{\prime \prime} \in \widetilde{M}_{\mathbb{Z}}\left(\Lambda_{\mathbf{V}^{\prime \prime}}\right)$ then $f^{\prime} * f^{\prime \prime} \in \widetilde{M}_{\mathbb{Z}}\left(\Lambda_{\mathrm{V}}\right)$ in the setup of (6.1). Thus $\kappa_{\nu}\left(\mathcal{U}_{\mathbb{Z}, \nu}^{+}\right) \subseteq \widetilde{M}_{\mathbb{Z}}\left(\Lambda_{\mathbf{V}^{\nu}}\right)$.

Let $\operatorname{Irr} \Lambda_{\mathrm{V}}$ denote the set of irreducible components of $\Lambda_{\mathrm{V}}$. The following proposition was conjectured by Lusztig and proved by him in the affine (and finite) case. The general case was proved by Kashiwara and Saito.

Proposition 6.3. For any $\nu \in\left(\mathbb{Z}_{\geq 0}\right)^{Q_{0}}$, we have $\operatorname{dim} \mathcal{U}_{\nu}^{+}=\# \operatorname{Irr} \Lambda_{\mathbf{V}^{\nu}}$

We then have the following important result due to Lusztig.

Theorem 6.4. Let $\nu \in\left(\mathbb{Z}_{\geq 0}\right)^{Q_{0}}$. Then 
(1) For any $Z \in \operatorname{Irr} \Lambda_{\mathbf{V}^{\nu}}$, there exists a unique $f_{Z} \in \kappa_{\nu}\left(\mathcal{U}_{\mathbb{Z}, \nu}^{+}\right)$such that $f_{Z}$ is equal to one on an open dense subset of $Z$ and equal to zero on an open dense subset of $Z^{\prime} \in \operatorname{Irr} \Lambda_{\mathbf{V}^{\nu}}$ for all $Z^{\prime} \neq Z$.

(2) $\left\{f_{Z} \mid Z \in \operatorname{Irr} \Lambda_{\mathbf{V}^{\nu}}\right\}$ is a $\mathbb{Q}$-basis of $\kappa_{\nu}\left(\mathcal{U}_{\nu}^{+}\right)$.

(3) $\kappa_{\nu}: \mathcal{U}_{\nu}^{+} \rightarrow \kappa_{\nu}\left(\mathcal{U}_{\nu}^{+}\right)$is an isomorphism.

(4) Define $[Z] \in \mathcal{U}_{\nu}^{+}$by $\kappa_{\nu}([Z])=f_{Z}$. Then $B_{\nu}=\left\{[Z] \mid Z \in \operatorname{Irr} \Lambda_{\mathbf{V}^{\nu}}\right\}$ is a $\mathbb{Q}$-basis of $\mathcal{U}_{\nu}^{+}$.

(5) $\kappa_{\nu}\left(\mathcal{U}_{\mathbb{Z}, \nu}^{+}\right)=\kappa_{\nu}\left(\mathcal{U}_{\nu}^{+}\right) \cap \widetilde{M}_{\mathbb{Z}}\left(\Lambda_{\mathbf{V}^{\nu}}\right)$.

(6) $B_{\nu}$ is a $\mathbb{Z}$-basis of $\mathcal{U}_{\mathbb{Z}, \nu}^{+}$.

From this theorem, we see that $B=\sqcup_{\nu} B_{\nu}$ is a $\mathbb{Q}$-basis of $\mathcal{U}^{+}$, which is called the semicanonical basis. This basis has many remarkable properties. One of these properties is as follows. Via the algebra involution of the entire universal enveloping algebra $\mathcal{U}$ of $\mathfrak{g}$ given on the Chevalley generators by $e_{i} \mapsto f_{i}$, $f_{i} \mapsto e_{i}$ and $h \mapsto-h$ for $h$ in the Cartan subalgebra of $\mathfrak{g}$, one obtains from the results of this section a semicanonical basis of $\mathcal{U}^{-}$, the lower half of the universal enveloping algebra of $\mathfrak{g}$. For any irreducible highest weight integrable representation $V$ of $\mathcal{U}$ (or, equivalently, $\mathfrak{g}$ ), let $v \in V$ be a non-zero highest weight vector. Then the set

$$
\{b v \mid b \in B, b v \neq 0\}
$$

is a $\mathbb{Q}$-basis of $V$, called the semicanonical basis of $V$. Thus the semicanonical basis of $\mathcal{U}^{-}$is simultaneously compatible will all irreducible highest weight integrable modules. There is also a way to define the semicanonical basis of a representation directly in a geometric way. This is the subject of the next subsection.

One can also obtain a geometric realization of the upper part $U^{+}$of the quantum group in a similar manner using perverse sheaves instead of constructible functions. This construction yields the canonical basis which also has many remarkable properties and is closely related to the theory of crystal bases.

6.2. Nakajima's Quiver Varieties. We introduce here a description of the quiver varieties first presented by Nakajima. They yield a geometric realization of the irreducible highest weight representations of a simply-laced Kac-Moody algebra.

Definition 6.5. For $\mathbf{v}, \mathbf{w} \in \mathbb{Z}_{\geq 0}^{I}$, choose I-graded vector spaces $\mathbf{V}$ and $\mathbf{W}$ of graded dimensions $\mathbf{v}$ and $\mathbf{w}$ respectively. Then define

$$
\Lambda \equiv \Lambda(\mathbf{v}, \mathbf{w})=\Lambda_{\mathbf{V}} \times \sum_{i \in I} \operatorname{Hom}\left(\mathbf{V}_{i}, \mathbf{W}_{i}\right) .
$$

Definition 6.6. Let $\Lambda^{s t}=\Lambda(\mathbf{v}, \mathbf{w})^{\text {st }}$ be the set of all $(x, t) \in \Lambda(\mathbf{v}, \mathbf{w})$ satisfying the following condition: If $\mathbf{S}=\left(\mathbf{S}_{i}\right)$ with $\mathbf{S}_{i} \subset \mathbf{V}_{i}$ is x-stable and $t_{i}\left(\mathbf{S}_{i}\right)=0$ for $i \in I$, then $\mathbf{S}_{i}=0$ for $i \in I$.

The group $G_{\mathbf{V}}$ acts on $\Lambda(\mathbf{v}, \mathbf{w})$ via

$$
(g,(x, t)) \mapsto\left(\left(g_{h(\rho)} x_{\rho} g_{t(\rho)}^{-1}\right),\left(t_{i} g_{i}^{-1}\right)\right) .
$$

and the stabilizer of any point of $\Lambda(\mathbf{v}, \mathbf{w})^{\text {st }}$ in $G_{\mathbf{V}}$ is trivial. We then make the following definition.

Definition 6.7. Let $\mathcal{L} \equiv \mathcal{L}(\mathbf{v}, \mathbf{w})=\Lambda(\mathbf{v}, \mathbf{w})^{s t} / G_{\mathbf{V}}$.

Let $\mathbf{w}, \mathbf{v}, \mathbf{v}^{\prime}, \mathbf{v}^{\prime \prime} \in \mathbb{Z}_{>0}^{I}$ be such that $\mathbf{v}=\mathbf{v}^{\prime}+\mathbf{v}^{\prime \prime}$. Consider the maps

$\Lambda\left(\mathbf{v}^{\prime \prime}, \mathbf{0}\right) \times \Lambda\left(\mathbf{v}^{\prime}, \mathbf{w}\right) \stackrel{p_{1}}{\longleftarrow} \tilde{\mathbf{F}}\left(\mathbf{v}, \mathbf{w} ; \mathbf{v}^{\prime \prime}\right) \stackrel{p_{2}}{\rightarrow} \mathbf{F}\left(\mathbf{v}, \mathbf{w} ; \mathbf{v}^{\prime \prime}\right) \stackrel{p_{3}}{\longrightarrow} \Lambda(\mathbf{v}, \mathbf{w})$,

where the notation is as follows. A point of $\mathbf{F}\left(\mathbf{v}, \mathbf{w} ; \mathbf{v}^{\prime \prime}\right)$ is a point $(x, t) \in \Lambda(\mathbf{v}, \mathbf{w})$ together with an $I$-graded, $x$-stable subspace $\mathbf{S}$ of $\mathbf{V}$ such that $\operatorname{dim} \mathbf{S}=\mathbf{v}^{\prime}=\mathbf{v}-\mathbf{v}^{\prime \prime}$. A point of $\tilde{\mathbf{F}}\left(\mathbf{v}, \mathbf{w} ; \mathbf{v}^{\prime \prime}\right)$ is a point $(x, t, \mathbf{S})$ of $\mathbf{F}\left(\mathbf{v}, \mathbf{w} ; \mathbf{v}^{\prime \prime}\right)$ together with a collection of isomorphisms $R_{i}^{\prime}: \mathbf{V}_{i}^{\prime} \cong \mathbf{S}_{i}$ and $R_{i}^{\prime \prime}$ : $\mathbf{V}_{i}^{\prime \prime} \cong \mathbf{V}_{i} / \mathbf{S}_{i}$ for each $i \in I$. Then we define $p_{2}\left(x, t, \mathbf{S}, R^{\prime}, R^{\prime \prime}\right)=(x, t, \mathbf{S}), p_{3}(x, t, \mathbf{S})=(x, t)$ and $p_{1}\left(x, t, \mathbf{S}, R^{\prime}, R^{\prime \prime}\right)=\left(x^{\prime \prime}, x^{\prime}, t^{\prime}\right)$ where $x^{\prime \prime}, x^{\prime}, t^{\prime}$ are determined by

$$
\begin{aligned}
R_{h(\rho)}^{\prime} x_{\rho}^{\prime} & =x_{\rho} R_{t(\rho)}^{\prime}: \mathbf{V}_{t(\rho)}^{\prime} \rightarrow \mathbf{S}_{h(\rho)}, \\
t_{i}^{\prime} & =t_{i} R_{i}^{\prime}: \mathbf{V}_{i}^{\prime} \rightarrow \mathbf{W}_{i} \\
R_{h(\rho)}^{\prime \prime} x_{\rho}^{\prime \prime} & =x_{\rho} R_{t(\rho)}^{\prime \prime}: \mathbf{V}_{t(\rho)}^{\prime \prime} \rightarrow \mathbf{V}_{h(\rho)} / \mathbf{S}_{h(\rho)} .
\end{aligned}
$$

It follows that $x^{\prime}$ and $x^{\prime \prime}$ are nilpotent.

Lemma 6.8. One has

$\left(p_{3} \circ p_{2}\right)^{-1}\left(\Lambda(\mathbf{v}, \mathbf{w})^{s t}\right) \subset p_{1}^{-1}\left(\Lambda\left(\mathbf{v}^{\prime \prime}, \mathbf{0}\right) \times \Lambda\left(\mathbf{v}^{\prime}, \mathbf{w}\right)^{s t}\right)$.

Thus, we can restrict (6.2) to $\Lambda^{\text {st }}$, forget the $\Lambda\left(\mathbf{v}^{\prime \prime}, \mathbf{0}\right)$-factor and consider the quotient by $G_{\mathbf{V}}$ and $G_{\mathbf{V}^{\prime}}$. This yields the diagram

$$
\mathcal{L}\left(\mathbf{v}^{\prime}, \mathbf{w}\right) \stackrel{\pi_{1}}{\leftarrow} \mathcal{F}\left(\mathbf{v}, \mathbf{w} ; \mathbf{v}-\mathbf{v}^{\prime}\right) \stackrel{\pi_{2}}{\longrightarrow} \mathcal{L}(\mathbf{v}, \mathbf{w}),
$$

where

$$
\mathcal{F}\left(\mathbf{v}, \mathbf{w}, \mathbf{v}-\mathbf{v}^{\prime}\right)
$$

$\stackrel{\text { def }}{=}\left\{(x, t, \mathbf{S}) \in \mathbf{F}\left(\mathbf{v}, \mathbf{w} ; \mathbf{v}-\mathbf{v}^{\prime}\right) \mid(x, t) \in \Lambda(\mathbf{v}, \mathbf{w})^{\text {st }}\right\} / G_{\mathbf{V}}$.

Let $M(\mathcal{L}(\mathbf{v}, \mathbf{w}))$ be the vector space of all constructible functions on $\mathcal{L}(\mathbf{v}, \mathbf{w})$. Then define maps

$$
\begin{gathered}
h_{i}: M(\mathcal{L}(\mathbf{v}, \mathbf{w})) \rightarrow M(\mathcal{L}(\mathbf{v}, \mathbf{w})) \\
e_{i}: M(\mathcal{L}(\mathbf{v}, \mathbf{w})) \rightarrow M\left(\mathcal{L}\left(\mathbf{v}-\mathbf{e}^{i}, \mathbf{w}\right)\right) \\
f_{i}: M\left(\mathcal{L}\left(\mathbf{v}-\mathbf{e}^{i}, \mathbf{w}\right)\right) \rightarrow M(\mathcal{L}(\mathbf{v}, \mathbf{w})) .
\end{gathered}
$$


by

$$
\begin{gathered}
h_{i} f=u_{i} f, \\
e_{i} f=\left(\pi_{1}\right) !\left(\pi_{2}^{*} f\right), \\
f_{i} g=\left(\pi_{2}\right) !\left(\pi_{1}^{*} g\right) .
\end{gathered}
$$

Here

$$
\mathbf{u}={ }^{t}\left(u_{0}, \ldots, u_{n}\right)=\mathbf{w}-C \mathbf{v}
$$

where $C$ is the Cartan matrix of $\mathfrak{g}$ and we are using diagram (6.3) with $\mathbf{v}^{\prime}=\mathbf{v}-\mathbf{e}^{i}$ where $\mathbf{e}^{i}$ is the vector whose components are given by $\mathbf{e}_{j}^{i}=\delta_{i j}$.

Now let $\varphi$ be the constant function on $\mathcal{L}(\mathbf{0}, \mathbf{w})$ with value 1 . Let $L(\mathbf{w})$ be the vector space of functions generated by acting on $\varphi$ with all possible combinations of the operators $f_{i}$. Then let $L(\mathbf{v}, \mathbf{w})=$ $M(\mathcal{L}(\mathbf{v}, \mathbf{w})) \cap L(\mathbf{w})$.

Proposition 6.9. The operators $e_{i}, f_{i}, h_{i}$ on $L(\mathbf{w})$ provide it with the structure of the irreducible highest weight integrable representation of $\mathfrak{g}$ with highest weight $\sum_{i \in Q_{0}} \mathbf{w}_{i} \omega_{i}$. Each summand of the decomposition $L(\mathbf{w})=\bigoplus_{\mathbf{v}} L(\mathbf{v}, \mathbf{w})$ is a weight space with weight $\sum_{i \in Q_{0}} \mathbf{w}_{i} \omega_{i}-\mathbf{v}_{i} \alpha_{i}$. Here the $\omega_{i}$ and $\alpha_{i}$ are the fundamental weights and simple roots of $\mathfrak{g}$ respectively.

Let $Z \in \operatorname{Irr} \mathcal{L}(\mathbf{v}, \mathbf{w})$ and define a linear map $T_{Z}$ : $L(\mathbf{v}, \mathbf{w}) \rightarrow \mathbb{C}$ that associates to a constructible function $f \in L(\mathbf{v}, \mathbf{w})$ the (constant) value of $f$ on a suitable open dense subset of $Z$. The fact that $L(\mathbf{v}, \mathbf{w})$ is finite-dimensional allows us to take such an open set on which any $f \in L(\mathbf{v}, \mathbf{w})$ is constant. So we have a linear map

$$
\Phi: L(\mathbf{v}, \mathbf{w}) \rightarrow \mathbb{C}^{\operatorname{Irr} \mathcal{L}(\mathbf{v}, \mathbf{w})} .
$$

Then we have the following proposition.

Proposition 6.10. The map $\Phi$ is an isomorphism; for any $Z \in \operatorname{Irr} \mathcal{L}(\mathbf{v}, \mathbf{w})$, there is a unique function $g_{Z} \in L(\mathbf{v}, \mathbf{w})$ such that for some open dense subset $O$ of $Z$ we have $\left.g_{Z}\right|_{O}=1$ and for some closed $G_{\mathbf{V}}$-invariant subset $K \subset \mathcal{L}(\mathbf{v}, \mathbf{w})$ of dimension $<\operatorname{dim} \mathcal{L}(\mathbf{v}, \mathbf{w})$ we have $g_{Z}=0$ outside $Z \cup K$. The functions $g_{Z}$ for $Z \in \operatorname{Irr} \Lambda(\mathbf{v}, \mathbf{w})$ form a basis of $L(\mathbf{v}, \mathbf{w})$.

\section{Further READing}

We have given here a brief overview of some topics related to finite-dimensional algebras and quivers. There is much more to be found in the literature. For basics on associative algebras and their representations, the reader may consult introductory texts on abstract algebra such as [5]. For further results (and their proofs) on Ringel-Hall algebras see the papers 14, 15, 16, 17, 18, of Ringel and Green's paper 2] and the references cited therein. The reader interested in species, which extend many of these results to non-simply-laced Lie algebras, should consult [1].

The book [6] covers the quiver varieties of Lusztig and canonical bases. Canonical bases are closely related to crystal bases and crystal graphs (see [3] for an overview of these topics). In fact, the set of irreducible components of the quiver varieties of Lusztig and Nakajima can be endowed with the structure of a crystal graph in a purely geometric way (see [4, 19]). Many results on Nakajima's quiver varieties can be found in the original papers [9, 11]. The overview article [10] is also useful.

Quiver varieties can also be used to give geometric realizations of tensor products of representations (see [7, 8, 13, 20]) and finite-dimensional representations of quantum affine Lie algebras (see [12]). This is just a select few of the many applications of quiver varieties. Much more can be found in the literature.

\section{REFERENCES}

[1] V. Dlab and C. M. Ringel. Indecomposable representations of graphs and algebras. Mem. Amer. Math. Soc., $6(173): \mathrm{v}+57,1976$.

[2] J. A. Green. Hall algebras, hereditary algebras and quantum groups. Invent. Math., 120(2):361-377, 1995.

[3] J. Hong and S.-J. Kang. Introduction to quantum groups and crystal bases, volume 42 of Graduate Studies in Mathematics. American Mathematical Society, Providence, RI, 2002.

[4] M. Kashiwara and Y. Saito. Geometric construction of crystal bases. Duke Math. J., 89(1):9-36, 1997.

[5] S. Lang. Algebra, volume 211 of Graduate Texts in Mathematics. Springer-Verlag, New York, 2002.

[6] G. Lusztig. Introduction to quantum groups, volume 110 of Progress in Mathematics. Birkhäuser Boston Inc., Boston, MA, 1993.

[7] A. Malkin. Tensor product varieties and crystals: GL case. Trans. Amer. Math. Soc., 354(2):675-704 (electronic), 2002.

[8] A. Malkin. Tensor product varieties and crystals: the ADE case. Duke Math. J., 116(3):477-524, 2003.

[9] H. Nakajima. Instantons on ALE spaces, quiver varieties, and Kac-Moody algebras. Duke Math. J., 76(2):365-416, 1994.

[10] H. Nakajima. Varieties associated with quivers. In Representation theory of algebras and related topics (Mexico City, 1994), volume 19 of CMS Conf. Proc., pages 139157. Amer. Math. Soc., Providence, RI, 1996.

[11] H. Nakajima. Quiver varieties and Kac-Moody algebras. Duke Math. J., 91(3):515-560, 1998.

[12] H. Nakajima. Quiver varieties and finite-dimensional representations of quantum affine algebras. J. Amer. Math. Soc., 14(1):145-238 (electronic), 2001.

[13] H. Nakajima. Quiver varieties and tensor products. Invent. Math., 146(2):399-449, 2001.

[14] C. M. Ringel. Hall algebras. In Topics in algebra, Part 1 (Warsaw, 1988), volume 26 of Banach Center Publ., pages 433-447. PWN, Warsaw, 1990. 
[15] C. M. Ringel. Hall algebras and quantum groups. Invent. Math., 101(3):583-591, 1990.

[16] C. M. Ringel. Hall algebras revisited. In Quantum deformations of algebras and their representations (RamatGan, 1991/1992; Rehovot, 1991/1992), volume 7 of Israel Math. Conf. Proc., pages 171-176. Bar-Ilan Univ., Ramat Gan, 1993.

[17] C. M. Ringel. The Hall algebra approach to quantum groups. In XI Latin American School of Mathematics (Spanish) (Mexico City, 1993), volume 15 of Aportaciones
Mat. Comun., pages 85-114. Soc. Mat. Mexicana, México, 1995.

[18] C. M. Ringel. Green's theorem on Hall algebras. In Representation theory of algebras and related topics (Mexico City, 1994), volume 19 of CMS Conf. Proc., pages 185245. Amer. Math. Soc., Providence, RI, 1996.

[19] Y. Saito. Crystal bases and quiver varieties. Math. Ann., 324(4):675-688, 2002.

[20] A. Savage. The tensor product of representations of $U_{q}\left(\mathfrak{s l}_{2}\right)$ via quivers. Adv. Math., 177(2):297-340, 2003.

Fields Institute and University of Toronto, Toronto, Ontario, Canada

E-mail address: alistair.savage@aya.yale.edu 